\title{
Kinetic Simulations of Nonequilibrium Plasmas
}

\author{
Alexandre Likhanskii ${ }^{\mathrm{a}}$ \\ ${ }^{a}$ Tech-X Corporation, 5621 Arapahoe Ave, Suite A, Boulder, CO 80031
}

\begin{abstract}
The paper presents study of one of the examples of nonequilibrium plasmas - dielectric barrier discharge in atmospheric air - using kinetic particle-in-cell code VORPAL. It has been demonstrated that the use of state-ofthe-art methods for solution of the electrostatic problems and the concept of variable-weight particles for reduction number of particles in dense plasma regions made simulations numerically efficient. Using the developed model the propagation of the cathode-directed streamer driven by $4 \mathrm{~ns}$ positive pulse has been analyzed in both 2D and 3D. The model reproduced all essential physical phenomena of the streamer propagation. The model has been validated via grid resolution study and study of the concept of variable-weight particles.
\end{abstract}

Keywords: nonequilibrium plasma, gas discharge, streamers, plasma aerodynamics

PACS: 52.25.Dg, 52.30.Ex, 52.40.Kh, 52.80.Tn

\section{INTRODUCTION}

Dielectric barrier discharge (DBD) plasma actuators have been widely studied both experimentally and numerically [1-9]. First successful flow separation control experiments were performed by Roth et al [1] using DBD plasma actuators, driven by low-frequency sinusoidal signal. Several research groups used similar DBD driving voltage and performed parametric study of the DBD input and output parameters [2-3]. Princeton group used nanosecond pulses superimposed on a bias voltage to increase DBD produced thrust [4]. Starikovskii et al. used high-voltage nanosecond pulses to create thermal perturbations in boundary layer, enhancing mixing between lowspeed flow in boundary layer and high-speed free stream flow [5]. This approach led to the suppression of the flow separation at $\sim 150 \mathrm{~m} / \mathrm{s}$ free stream flow velocities. Theoretical estimates of the maximum DBD induced flow velocities show that DBD operation may be improved once the strong electric field in the discharges are achieved. One possible way to get strong electric field in DBD is to use high-voltage nanosecond pulses. However, the problem of reverse breakdown, which significantly reduces induced thrust, is still to be solved.

Both experimental results and theoretical studies show high potential of pulsed DBD plasma actuator. However, in order to improve of the pulsed DBDs, it is necessary to accurately resolve all corresponding physical phenomena. Numerical study of the DBD operation is conventionally based on two plasma modeling approaches - fluid and kinetic plasma description. Font et al. treated plasma kinetically using PIC-DSMC code and predicted Townsend type of the discharge [6]. Recently, majority of the groups used fluid approach (drift-diffusion approximation) to either describe the breakdown stage of the discharge or plasma decay stage [7-9]. These modeling efforts resulted in the qualitative description of the force generation by the DBD plasma actuator at atmospheric pressures. However, the obtained results still lack validation against experimental data. Recently, Jon Poggie compared two fluid approaches - 5-moment model (more accurate model) and 2-moment model (simplified model) for the gas discharge simulation [10]. The models showed significant differences in electron velocities and electron temperatures in gas discharge.

\section{PHYSICAL AND NUMERICAL MODEL}

The dielectric barrier discharge plasma actuator consists of two electrodes, separated by the dielectric (Fig. 1). Once the voltage is applied between the electrodes, the plasma is generated near the edge of the exposed electrode and spreads along dielectric surface. 


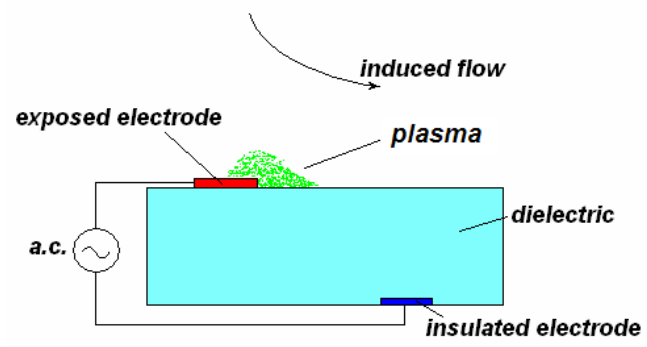

FIGURE 1. DBD plasma actuator.

The modeling of the DBD plasma actuator has been performed using 2D/3D Particle-In-Cell (PIC) code VORPAL [11]. The modeled gas was air with simplified chemistry. Generated weakly-ionized plasma was modeled as five-species mixture, consisting of neutral molecular oxygen (fluid), neutral molecular nitrogen (fluid), positive molecular oxygen ions (kinetic), positive molecular nitrogen ions (kinetic) and electrons (kinetic). Only three types of collisions - elastic collision, excitation and ionization - were taken into account for the breakdown modeling. Data on the cross-sections was taken from Ref [12-13]. This assumption is justified since the breakdown time scales are of the order of nanoseconds, and characteristic time scales of remaining important plasma processes, such as recombination, attachment and detachment are much longer. The solver for the DBD problem consisted of two major parts - Poisson Solver for electric potential and electric field distribution and Boris push for charged particles motion.

Poisson equation was solved using the biconjugate gradient method with algebraic multigrid preconditioner. The solution of Poisson Solver was considered converged when the norm of relative residual became less than $10^{-4}$. The convergence was achieved after 2-3 iterations per time step, compared to hundreds of iterations for widely used Successive Overrelaxation Method for the same problem.

Charged particles were represented via macroparticles, i.e. particles, consisting of several real particles. In order to define the number of real particles in macroparticle, VORPAL uses the concept of nominal density of macroparticles. The nominal density of macroparticles is the plasma density in the simulation domain when each numerical cell contains one macroparticle. For example, in 3D DBD simulations, considered below, the nominal density of macroparticles was $3 * 10^{17} \mathrm{~m}^{-3}$ and the cell size was $2 \times 2 \times 2$ microns, leading to 2.4 charged particles per macroparticle. The choice of the concept of nominal number density becomes clear in 2D, when the macroparticle represents not the absolute number of charged particles, but the number of charged particles per unit length.

One of the major obstacles for modeling atmospheric gas discharges using PIC codes is the exponential growth of the number of particles in the plasma regions with relatively high charged particles density, such as streamer channel. For example, in the above mentioned case of 3D DBD simulation, one needs to use $10^{4}$ macroparticles per cell in order to represent streamer body with plasma density of $3 * 10^{21} \mathrm{~m}^{-3}$. Such large number of similar charged particles significantly reduces the computational speed, making PIC codes unfeasible for discharge problem. In VORPAL this problem is solved using the concept of variable-weight (VW) particles. In this concept, macroparticle has not only defined position and velocity, but also a "weight", which defines the number of standard macroparticles in this particular VW macroparticle. Let's consider the above described example for 3D DBD, where one standard macroparticle consisted of 2.4 electrons. If we assign weight $\mathrm{W}=2$ to the $\mathrm{VW}$ macroparticle, it will consist of two standard macroparticles or 4.8 electrons, and so on. This concept is useful for the description of atmospheric gas discharges. For example, in the streamer body particles barely move, since the electric field is quite weak there. Therefore, without loss of generality, many of these particles can be combined into particles with higher weight. Depending on the problem, we can use different algorithms for this combination. For example, if one considers ions during the breakdown and want to treat them just as charge background, all ions within a cell can be combined into one large macroparticle (since ions barely move during the breakdown). For the electrons, one might need to have several particles within a cell in order to reproduce local temperature distribution. In order to accomplish both these approaches, one can use threshold number of particles within a cell, i.e. the macroparticles are combined only when the number of macroparticles inside the cell exceeds the threshold limit. In order to avoid generation of extremely large macroparticles, we also limit the maximum allowed weight.

One of the key elements for the description of the streamer propagation is the presence of electron source in front of the streamer head. This source is due to the photoionization, occurring around regions of high electric field and high electron concentrations. Unfortunately, VORPAL does not have a photoinization model at this moment. In order to account for it, a few macroparticles in the computational domain 100 microns above the DBD surface at 
randomly loaded at each time step. On one hand, this source of particles is relatively small compared to the generated plasma. On the other hand, it is sufficient to provide electrons in order to support streamer propagation.

The grid in the simulation domain was chosen based on the typical plasma scales and typical length of mean free path for electrons. Since maximum cross-sections in the model were of the order of $10^{-20} \mathrm{~m}^{2}$, and neutral gas number density is $\sim 2.5^{*} 10^{25} \mathrm{~m}^{-3}$, the minimum mean free path for the electrons is of the order of microns. From fluid codes [7], typical plasma sheath scales are $\sim 10$ microns. Therefore, the grid size was chosen to be from 0.5 to 2 microns, depending on the problem. The time step was chosen to be 0.15 picoseconds in order to resolve all physical processes.

\section{SIMULATION RESULTS}

\section{D Simulations}

For 2D simulations the following DBD parameters were chosen. The lengths of the exposed and grounded electrodes were 0.2 and $1.6 \mathrm{~mm}$ correspondingly. The width of the exposed electrode was 20 microns and the width of grounded electrode was 40 microns. Width of the dielectric was 100 microns and its relative dielectric permittivity was equal to 1 . The simulation domain was $2 \times 1 \mathrm{~mm}$. The grid size was $1 \mathrm{x} 1 \mu \mathrm{m}$. The nominal density of macroparticles was equal to $10^{17} \mathrm{~m}^{-3}$. The DBD driving voltage was $3 \mathrm{kV} 4 \mathrm{~ns}$ positive pulse. For the sorting algorithm in order to combine particles within one cell, the following parameters were used. Particles were combined, when the number of particles in a cell exceed threshold limit equal to 10.

Let us consider the discharge development. The simulations started with no plasma, and the electric potential distribution was defined only by voltage at electrodes (Fig. 2(a)). Once the voltage between the electrodes increases, the plasma was generated near the edge of exposed electrode. Since this plasma is highly-conductive, its potential becomes equal to the potential of the exposed electrode (Fig. 2(b)). Once the streamer propagates along the dielectric, it carries the potential of the exposed electrode (Fig. 2(c)). Electron distribution in the streamer is shown in Figure 3. Electron concentration is highest in the streamer body.

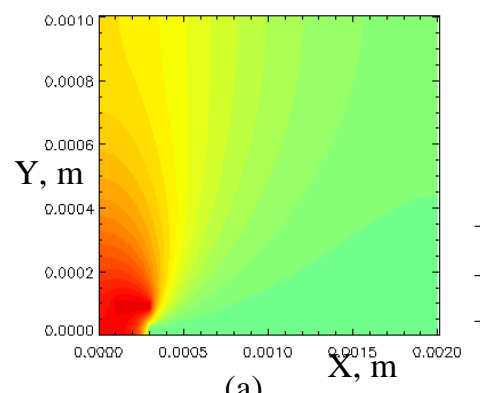

(a)

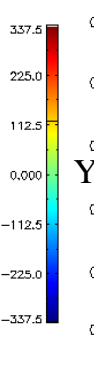

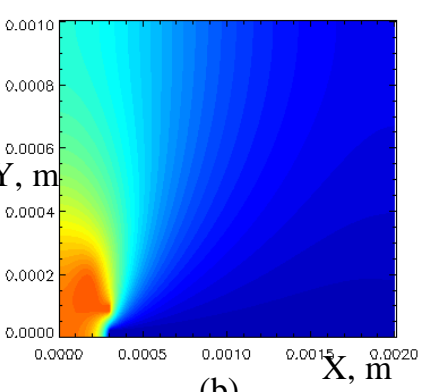

(b)

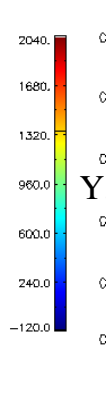

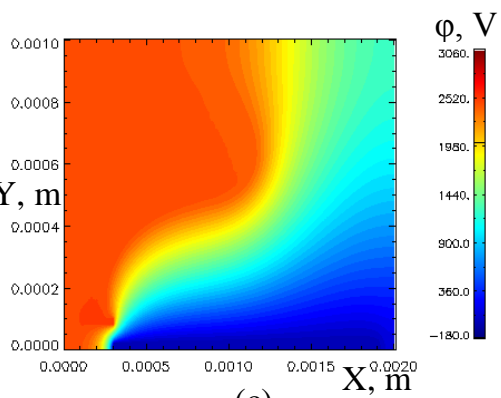

(c)

FIGURE 2. Electric potential evolution during the streamer formation. DBD is driven by $3 \mathrm{kV} 4 \mathrm{~ns}$ positive pulse. (a)

Corresponds to the initial stage of the DBD discharge (0.3 ns after the start of the pulse),

(b) shows the start of the streamer propagation ( $2.1 \mathrm{~ns}$ after the start of the pulse),

(c) shows the generated streamer ( $3 \mathrm{~ns}$ after the start of the pulse).

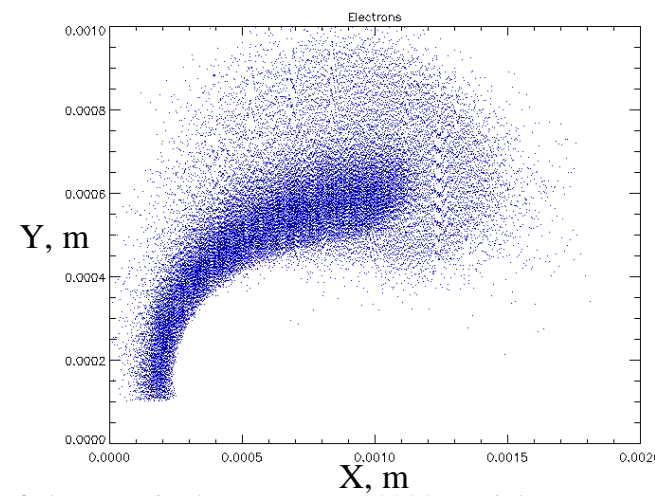

FIGURE 3. Distribution of electrons in the streamer (20000 particles were randomly chosen for this plot). 
Figures 3 and 4 demonstrate the concept of variable-weight particles. Initially, all macroparticles have weight equal to 1 (see above section for description). Once the plasma is generated and number of macroparticles inside the cell exceed the threshold limit (10 macroparticles per cell), which is true in streamer body, particles are combined into larger macroparticles. One can notice from Fig. 3 that dense streamer body starts at $\mathrm{x} \approx 0.0001 \mathrm{~m}$ and ends at $\mathrm{x} \approx 0.0011 \mathrm{~m}$. Figure 4 shows the highest concentration of electrons with largest weight right in this region. However, in front of the streamer head, electrons are rarely combined, and the avalanche ionization is resolved with high accuracy.

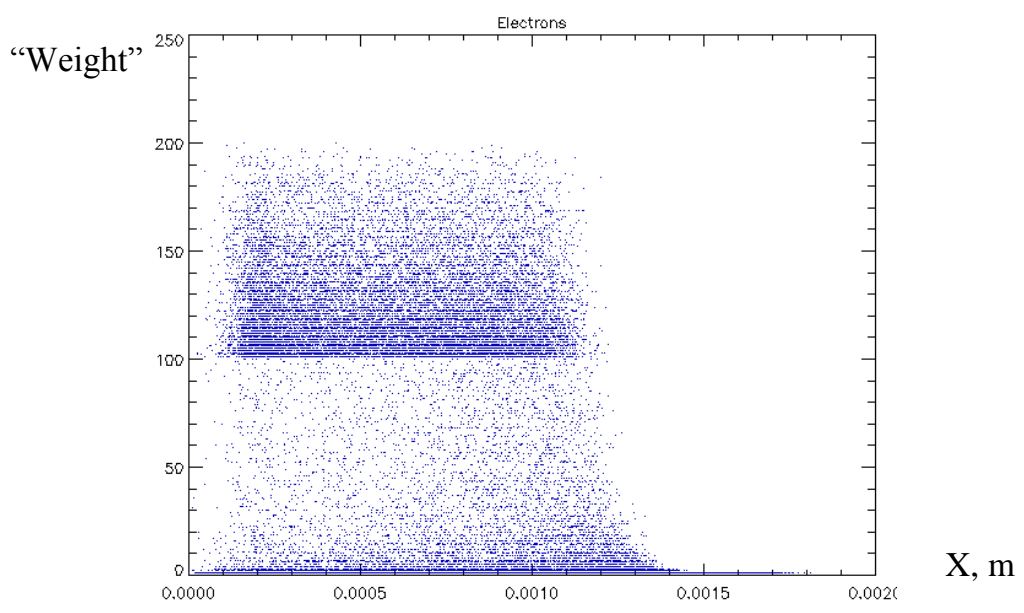

FIGURE 4. Distribution of variable-weight particles.

Macroparticles, representing electrons, are combined into large macroparticles in the regions of high electrons concentration (streamer body) and are not combined in the regions of low electron concentration (in front of the streamer head).

\section{Validation Study}

In order to verify the accuracy of the obtained results, the grid resolution study was performed. For the additional simulations, the grid size of $0.5 \times 0.5$ microns was chosen. The time step remained the same. The number of particles per macroparticle was not changed. The number particle loads at each time step was also constant. However, the threshold for combining macroparticles was changed from 10 to 3 . The reason for this change is the following. On the coarse grid, particles started combining when there were 10 particles in the $1 \times 1$ micron cell. If the same combining algorithm is applied to the fine grid, it would mean that particles start combining only when there are 10 particles per $0.5 \times 0.5$ micron cell (which is similar to combining particles in $1 \times 1$ micron cell with threshold equal to 40). Therefore, threshold equal to 3 (which is similar to 12 for $1 \times 1$ micron grid) was chosen for the fine grid. The results of the simulation of electric field are shown in Figs. 4(a,b) and 5(a,b). The major difference between the results is time of the breakdown. Similar streamers were developed after 2.7 and $3.3 \mathrm{~ns}$ after the pulse was applied for coarse and fine grid correspondingly. Another difference is in streamer height above the dielectric surface (400 and 460 microns above the surface). However, the streamer structure is rather similar and the rest of streamer characteristics are also similar. Figure 5(a,b) shows the comparison between distribution of $\mathrm{x}$-component of electric field in the y-cut, corresponding strongest electric field at the streamer tip. Both maximum electric field and e-field distribution are in good agreement.

Another validation study was performed for the concept of variable-weight particles. The main parameter for this concept is the threshold number of particles in the cell for particle combining. In order to study the influence of this threshold value on the streamer development, the simulation on the fine grid $(0.5 \times 0.5$ microns) was performed with threshold equal to 10 . The results of this simulation were compared against the results of the similar simulation with threshold equal to 3 (Figs 3(b,c) and 4(b,c)). The results are in good agreement. Electric field distribution is almost the same $3.3 \mathrm{~ns}$ after the start of the pulse. It is also worth mentioning that concept of variable-weight particles significantly reduces the computational time. For example, it took approximately 9 and 3.5 hours to compute $3.3 \mathrm{~ns}$ on the fine grid with thresholds equal to 10 and 3 correspondingly. Besides it, use of this concept significantly reduces RAM usage, which is usually a problem for PIC simulations with large number of particles. 


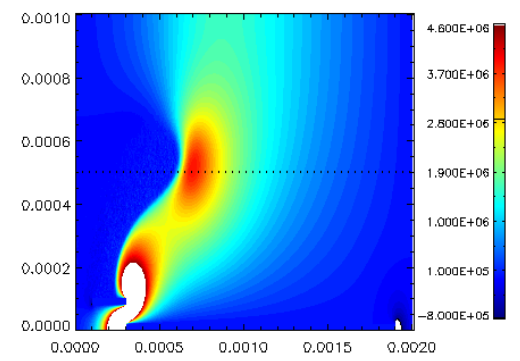

(a)

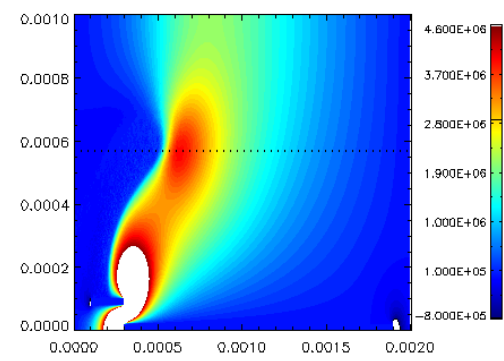

(b)

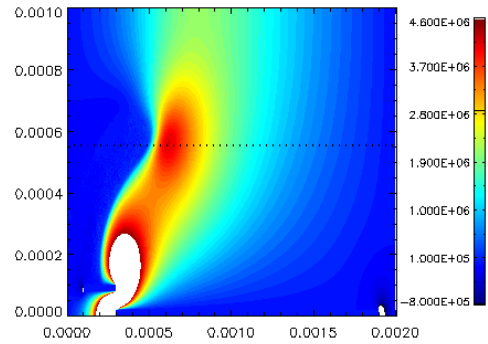

(c)

FIGURE 5. Distribution of $x$-component of electric field. DBD is driven by $3 \mathrm{kV} 4 \mathrm{~ns}$ positive pulse.

(a) $2.7 \mathrm{~ns}$ after the start of the pulse. Grid size is $1 \times 1$ micron. Threshold for VW particles is 10;

(b) $3.3 \mathrm{~ns}$ after the start of the pulse. Grid size is $0.5 \times 0.5$ microns. Threshold for VW particles is 3 ;

(c) $3.3 \mathrm{~ns}$ after the start of the pulse. Grid size is $0.5 \times 0.5$ microns. Threshold for VW particles is 10.

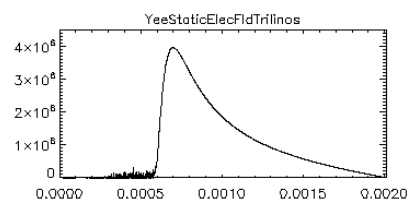

(a)

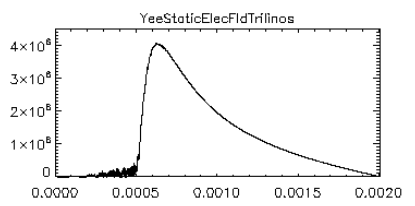

(b)

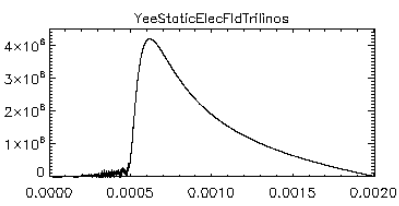

(c)

FIGURE 6. Distribution of $\mathrm{x}$-component of electric field at the $\mathrm{y}$-cut shown in figure 5 via dashed line. DBD is driven by $3 \mathrm{kV}$ 4 ns positive pulse.

(a) $2.7 \mathrm{~ns}$ after the start of the pulse. Grid size is $1 \mathrm{x} 1 \mathrm{micron}$. Threshold for VW particles is 10;

(b) $3.3 \mathrm{~ns}$ after the start of the pulse. Grid size is $0.5 \times 0.5$ microns. Threshold for VW particles is 3 ;

(c) $3.3 \mathrm{~ns}$ after the start of the pulse. Grid size is $0.5 \mathrm{x} 0.5$ microns. Threshold for VW particles is 10.

\section{D Simulations}

One of the open questions for the DBD operation is the formation of a filamentary structure perpendicular to the direction of streamer propagation. In order to resolve this structure, accurate 3D simulations of the DBD should be performed. In this section, the first attempt for these simulations using PIC approach is discussed. The geometry for the simulations was the following. The simulation domain was $1 \times 1 \times 0.5 \mathrm{~mm}$. The sizes of the exposed and grounded electrodes were $0.2 \times 0.02 \times 0.5 \mathrm{~mm}$ and $0.6 \times 0.04 \times 0.5 \mathrm{~mm}$ correspondingly. The dielectric thickness was equal to 0.1 $\mathrm{mm}$. Dielectric permittivity was equal to 1 . DBD was driven by $3 \mathrm{kV}$ 4ns positive pulses. Grid size was equal to $2 \times 2 \times 2$ microns. The threshold for combining ions (both nitrogen and oxygen) within a cell was equal to 1 (since ions can be considered steady during the breakdown). The standard macroparticle consisted of 2.4 particles (electrons, oxygen or nitrogen positive ions). The threshold for combining macroparticles within a cell was equal to 3 . It is worth pointing out, that the grid size and threshold were chosen based on the maximum RAM, available for the simulation on the 16 node (128 core) cluster (with 8 GB RAM for each node). Therefore, the results were not checked for numerical accuracy.

Figure 7-8 show the distributions of electrons, electric potential and $x$-component of electric field when the streamer is generated. The streamer profile and streamer's characteristics are similar to ones obtained in 2D simulations. The main difference is the speed of streamer generation ( $2.3 \mathrm{~ns}$ compared to 2.7 and $3.3 \mathrm{~ns})$. This difference is due to the non-sufficient spatial resolution of the simulations. The same effect was observed during the grid resolution study for 2D simulations. 


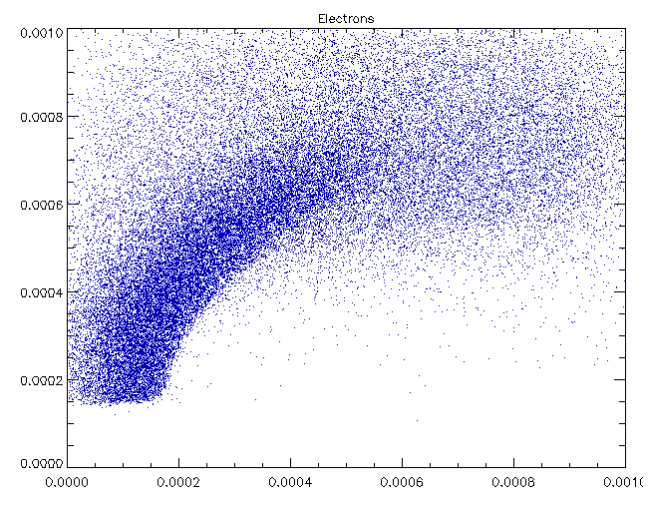

(a)

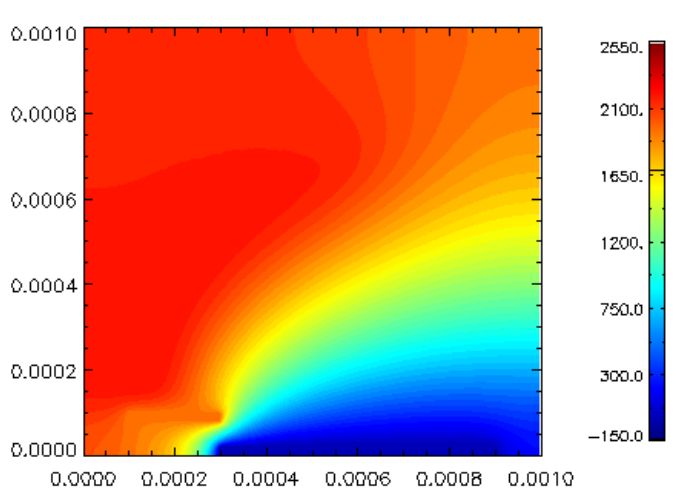

(b)

FIGURE 7. (a) Distribution of electrons in $x$-y plane (only 20000 randomly chosen electrons are plotted);

(b) Distribution of electric potential.

DBD is driven by $3 \mathrm{kV} 4 \mathrm{~ns}$ positive pulse. $2.3 \mathrm{~ns}$ after the start of the pulse.

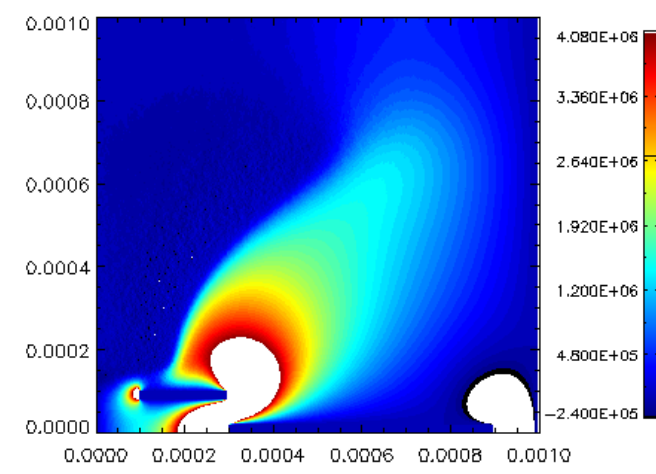

(a)

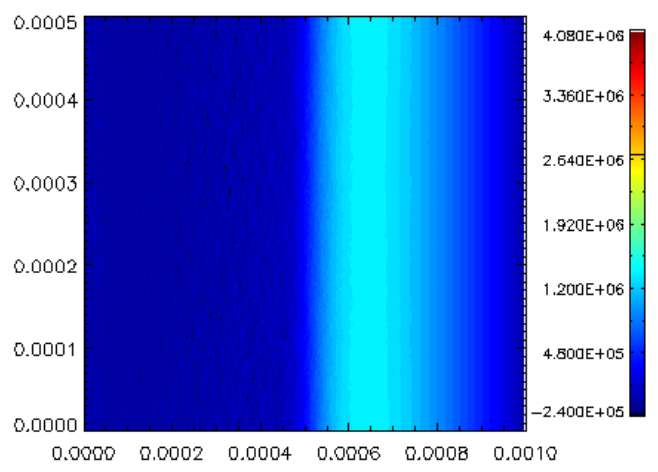

(b)

FIGURE 8. (a) Distribution of $x$-component of electric field in $x-y$ plane;

(b) Distribution of $\mathrm{x}$-component of electric field in $\mathrm{x}-\mathrm{z}$ plane (y-cut corresponds to streamer head).

$\mathrm{DBD}$ is driven by $3 \mathrm{kV} 4 \mathrm{~ns}$ positive pulse. $2.3 \mathrm{~ns}$ after the start of the pulse.

\section{CONCLUSIONS}

This paper presented results on the numerical simulations of discharge stage of pulse-driven DBD in atmospheric air in 2D and 3D using PIC approach. Unlike the conventional DBD modeling, charged particles were considered not as a continuous fluid, but as ensembles of real charged particles. This approach provides more accurate solution for the streamer generation problem. In order to significantly increase computational speed of the simulations, the state-of-the-art numerical techniques were used. The solution of Poisson Equation via biconjugate gradient method with algebraic multigrid preconditioner lead to 2 order of magnitude reduction in the number of iterations for the convergence, compared to conventional Successive Over-Relaxation Method. The use of variable-weight particles allowed combining macroparticles into large ones in the region of relatively dense plasma without loss of accuracy. It lead to several times faster simulations and reduction of required RAM.

DBD driven by $3 \mathrm{kV}, 4 \mathrm{~ns}$ positive pulses was modeled both in $2 \mathrm{D}$ and $3 \mathrm{D}$. The simulations resolved all essential physical phenomena of the streamer generation and propagation, such as formation of highly-conductive streamer channel, transfer of ionization region to the streamer head, etc. The streamer, generated using PIC approach was thicker and propagated higher above the surface, compared to the streamer, generated using fluid approach.

Validation study demonstrated the feasibility of the concept of variable-weight particles for the discharge description. The grid resolution study showed some discrepancy between speed of streamer generation of fine and coarse grid, however, the rest of streamer characteristics remained the same. 


\section{ACKNOWLEDGMENTS}

This work has been funded by NASA SBIR Phase I contract number NNX10CE78P, Technical Officer Dr. David E. Ashpis. Author would like to thank Drs. Mikhail Shneider, Sergey Macheret, David Ashpis, Peter Messmer, Peter Stoltz, Sudhakar Mahalingam, Yongjun Choi and Dmitry Opaits for fruitful discussions.

\section{REFERENCES}

1. R. Roth, Sherman and S. Wilkinson, AIAA Journal 38, 1166-1172 (2000).

2. M. Post and T. Corke, AIAA Journal 42, 2177-2184 (2004).

3. C. Enloe, T. McLaughlin, R. VanDyken, K. Kachner, E. Jumper, T. Corke, et al., AIAA Journal 42, 595-604 (2004).

4. D. Opaits, M. Shneider, R. Miles, A. Likhanskii and S. Macheret, Journal of Applied Physics, 104, 043304-043304-15 (2008).

5. M. Post and T. Corke, AIAA Journal 42, 2177-2184 (2004).

6. D. Roupassov, A. Nikipelov, M. Nudnova and S. Starikovskii, "Flow Separation Control by Plasma Actuator with Nanosecond Pulse Periodic Discharge", Proceedings of $46^{\text {th }}$ Aerospace Sciences Meeting and Exhibit, Reno, NV, 2008

7. G. Font, C. Enloe, T. McLaughlin and D. Orlov, "Plasma Discharge Characteristics and Experimentally Determined Boundary Conditions for a Plasma Actuator", Proceedings of $45^{\text {th }}$ AIAA Aerospace Meeting and Exhibit. Reno, NV, 2007

8. A. Likhanskii, M .Shneider, S. Macheret and R. Miles, Physics of Plasmas 14, 073501-073501-8 (2007).

9. J. Boeuf and L. Pitchford, Journal of Applied Physics 97, 103307-103307-10 (2005).

10. A. Hoskinson, "Measurements and Simulations of Surface Dielectric Barrier Discharges Used as Plasma Actuators", Ph.D. Thesis, University of Madison-Wisconsin, 2009

11. J. Poggie, "Role of Charged Particle Inertia in Pulsed Electrical Discharges", Proceedings of $48^{\text {th }}$ AIAA Aerospace Meeting and Exhibit, Orlando, Fl, 2010

12. C. Nieter and J. Cary, J. Comp. Phys, 196, 448-472 (2004).

13. Y. Itikawa et.al., J. Phys. Chem. Ref. Data, 15, 985-1010 (1986)

14. Y. Itikawa et.al., J. Phys. Chem. Ref. Data, 18, 23-42 (1989) 